\title{
Proposal for progressive loading of the hip abductors under mechanically unstable conditions: An electromyography study
}

\author{
Nejc Sarabon (1,2), Palma Polona (3), Samantha Burggraf (1)
}

(1) Ludwig Boltzmann Institute of Electrical Stimulation and Physical Rehabilitation, Vienna, Austria; (2) University of Primorska, Science and Research Centre, Koper, Slovenia; (3) University College of Health Care, University of Ljubljana, Ljubljana, Slovenia.

\begin{abstract}
The aim of this study was to test the effect of the stance width and asymmetry on muscle activation patterns during balancing on a tilt board. Eleven young healthy volunteers took part in the tests. After the standardized warm-up and customization protocol had been carried out, they balanced five times for 60 seconds on a tilt board, using a different foot position each time - (i) wide symmetrical, (ii) narrow symmetrical, (iii) moderate asymmetrical, (iv) extreme asymmetrical, and (v) single leg. Pair of electromyographic electrodes was glued over the gluteus medius muscle on both sides from which signals were acquired. The average values of the pre-processed signals were normalized and quantified. Repeated measures analysis of variance and t-tests revealed a systematic effect of the foot positions on the amount of the gluteus medius activation. Its activation was significantly increased in both asymmetrical stances when the foot was moved closer to the tilt board's axis of rotation and most prominently when the single leg stance was used. These results point out the importance of the foot positioning for the actual muscle function while balancing on a tilt board. We believe that different levels of feet positioning asymmetry should be used for gradual loading of the extremity and for provoking activity in hip side stabilizers.
\end{abstract}

Key Words: dynamic balance, hip stability, tilt board, asymmetry

European Journal Translational Myology - Basic Applied Myology 2010; 1 (4): 187-192

Closed kinetic chain exercises, which require a considerable level of pelvic stability, are an integral part of rehabilitation programs for treating lower extremity injuries. Recent investigations [2,4,9,18,26] have highlighted the importance of the muscles acting upon the hip joint, specifically the hip abductor muscles, in preventing and recovering from distal lower extremity injuries. The gluteus medius muscle (GM) is strongly involved in the control of hip joint stability [10] and is active when postural stability is challenged in the medio-lateral direction [22,24]. Activation levels of GM during lower extremity rehabilitation exercises have received considerable attention as investigators have tried to identify appropriate treatment strategies $[6,12,13,20,23,28]$. However, these studies have primarily focussed on isolated open kinetic chain exercises and have left a gap concerning the activation patterns of these muscles in functional closed kinetic chain activities.

Winter and colleagues [27] identified synergistic muscle activity at the hip as a fundamental mechanism for medio-lateral postural control during standing. Moreover Grelsamer and McConnell [11] stress the importance of training the GM while the patient is weight bearing in order to control the frontal orientation of the pelvis and concomitant internal rotation. Alterations in activation patterns of these muscles have been documented for patients suffering from low back, knee and ankle injuries [2,4,5]. Such changes were evidenced during diverse weight bearing activities like standing, responding to mechanical perturbations, chair rising and step climbing. The well documented role of hip abductors during activities of daily living $[17,19,21,25]$ supports the need for addressing the function of these muscles in rehabilitation and in the prevention of lower extremity injuries.

Ayotte and colleagues [1] evaluated and reported on differences in GM activity between various unilateral weight bearing exercises, such as squats and step-ups, thus providing evidence regarding the functioning of hip muscles during moderately demanding tasks. 
Hip abductors and unstable conditions

European Journal Translational Myology - Basic Applied Myology 2010; 1 (4): 187-192

Another study [8] has reported on GM activity during basic and also on more progressive/demanding rehabilitation exercises. However, only a few exercises used functional daily movements. None of them used combinations of weight bearing activity on unstable surfaces such as those required in common daily activities and especially those involved in sports activities.

The purpose of this study was to quantify and compare GM levels of activation during a series of balance tasks on an inherently unstable tilt platform, using different foot positions while standing either on both legs or on a single leg. The findings of this study will provide valuable information which will help in designing a progressive kinesiotherapeutic hip stability exercise programme.

\section{Materials and Methods}

\section{Subjects}

Eleven students of physical education ( 9 male, 2 female; age $24.4 \pm 3.1$ years; body height $172.7 \pm 6.3$ $\mathrm{cm}$; body weight $72.0 \pm 8.3 \mathrm{~kg}$ ) participated in the study. The study was carried out in accordance with Helsinki declaration. Prior to the enrolment, a detailed description of the experimental protocol was explained to the subjects. After they had signed the informed consent, the subjects went through a familiarization session during which they got accustomed to the tiltboard dynamic balance tasks that were used in the experiment later on.

\section{Measurements}

Before the start of the measurements, a standardized 15-minute warm-up was carried out $(6$ min running and hopping on the spot, $3 \mathrm{~min}$ stretching, $3 \mathrm{~min}$ muscle tonus enhancement, $3 \mathrm{~min}$ of progressive balancing tasks). Then, dual self-adhesive surface electrodes (Noraxon, Scottsdale, Arizona, USA) were placed over the medial gluteal muscles (GM) on both legs, for the purpose of recording electrophysiological activity of the muscle (EMG). The ground electrode was fixed on a patella. Before the electrodes were glued over the belly muscle the skin was prepared according to the SENIAM guidelines (shaving, abrasion and alcohol cleaning) so that a skin impedance of $<5 \mathrm{k} \Omega$ was achieved. The electrodes were connected to the central EMG unit (Myosystem 1400A, Noraxon, Scottsdale, Arizona, USA) placed in the proximity of the subject. The EMG signals were acquired at $4000 \mathrm{~Hz}$ and stored on a personal computer for later analyses.

As an initial test, each subject performed three repetitions of maximal voluntary contractions (MVC), each of three-seconds, with each leg. An isometric abduction in the side-lying position and at $10^{\circ}$ hip abduction was used. This was done with the aim of normalizing the GM EMG activity achieved during the balancing tasks that followed.

After this, the subject performed a set of five balancing tasks on a custom made inverted pendulum device, i.e. a tilt-platform (width $50 \mathrm{~cm}$, height $7 \mathrm{~cm}$ ) that was unstable in the frontal plane.

These balance tasks employed five different foot placements: (i) (WS) wide symmetrical stance during which the feet were $40 \mathrm{~cm}$ apart, each at a distance of $20 \mathrm{~cm}$ from the rotational axis of the platform, (ii) (NS) narrow symmetrical stance with the feet $20 \mathrm{~cm}$ apart, each at a $10 \mathrm{~cm}$ distance from the axis, (iii) (ME) moderate asymmetrical stance with the feet $34 \mathrm{~cm}$ apart, non-dominant leg $20 \mathrm{~cm}$ and dominant leg at 14 $\mathrm{cm}$ distance from the axis, (iv) (EA) extreme asymmetrical stance with the feet $27 \mathrm{~cm}$ apart, the nondominant leg $20 \mathrm{~cm}$ and dominant leg at $7 \mathrm{~cm}$ distance from axis, and (v) (SL) single leg stance on a dominant leg.

Each subject carried out two rounds of familiarization trials, each task lasting $30 \mathrm{~s}$, each round consisted five balancing tasks performed in random order. A third round, also in random order, this time for $60 \mathrm{~s}$, was performed for each of the 5 tests. Altogether each subject performed 15 trials, each of the 5 tasks 3 times, that is, twice for $30 \mathrm{~s}$ and once for $60 \mathrm{~s}$. Three-minute breaks were taken between each of the successive trials. The entire testing lasted $75 \mathrm{~min} /$ person. The familiarization trials were only used to get accustomed with the tasks, but only the $60 \mathrm{~s}$ trials were used for the further analyses.

\section{Analysis}

The stored signals were first zero aligned and bandpass filtered (2nd order Butterworth, $20 \mathrm{~Hz} / 1 \mathrm{kHz}$ ). Then, each signal was analysed in the frequency and amplitude domain for the whole 60-second interval. Root mean square (RMS) was calculated from the previously rectified and smoothed signal (flow arithmetic mean). Frequency analysis was done on Furrier transformed signals (Henning window) and the mean frequency of the power spectrum (MF) was calculated. The same processing and quantification was carried out for the MVC contractions at a one-second interval where the highest RMS was achieved.

For statistical analyses, SPSS 17 software (SPSS Inc., Chicago, USA) was used. RMS and MF were normalized and transformed to \%MVC values. Descriptive statistics were calculated and the results represented as mean \pm standard deviation. Repeated measures analysis of variance (RANOVA) was used to test potential differences in muscle activation between the balance tasks. Pairwise t-tests were applied for post hoc analysis. The level of statistical significance was set at 0.05 . 


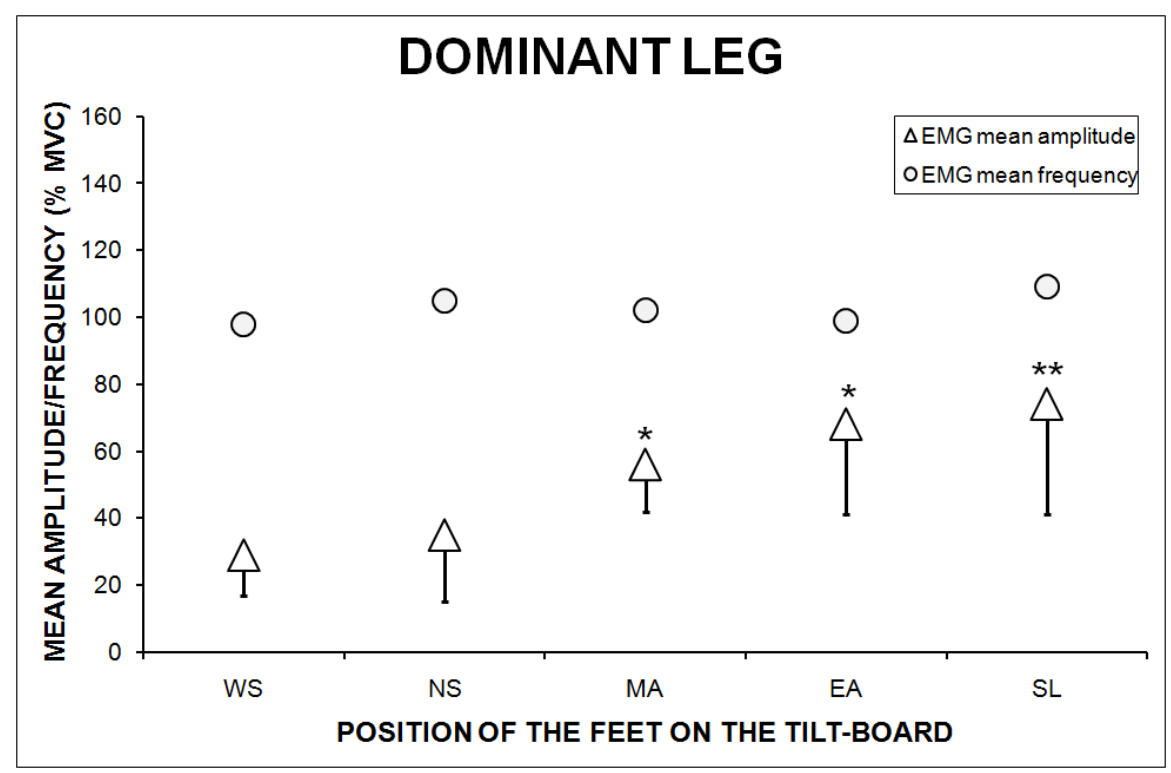

Fig 1. Results for the GM activity for the dominant leg during all the stances.

\section{Results}

Statistically significant differences among the five balance tasks (i.e. feet positions) were found in GM mean EMG amplitude in both the dominant and nondominant leg (RANOVA, $\mathrm{P}<0.05$ ). Pairwise comparisons revealed there was almost no change when subjects were using the NS stance instead of the WS stance (t-test, $\mathrm{p}<0.05 ; 29 \pm 12$ vs. $35 \pm 20 \%$ MVC). However, an almost linear progression in the mean EMG amplitude of the dominant leg was observed when bringing the same foot closer to the axis of the board (t-test relative to WS, $\mathrm{p}=0.048$ and $\mathrm{p}$ $=0.009$ for $\mathrm{MA}$ and EA, respectively) and even more when balancing on the dominant leg alone (WS/SL ttest, $p=0.001$ ) (see Figure 1). No systematic change could be seen in the frequency domain of the dominant leg (RANOVA, $p>0.05$ ). The values remained close to those of the side-lying hip abduction which was used as a normalization task (from $98 \pm 29$ to $109 \pm 20$ $\%$ of that in MVC).

Opposite to the dominant leg, the foot of the nondominant leg was held in the same place for WS, MA and EA balance tasks. In the SL task, the nondominant leg was held in the air. The results for the GM mean EMG amplitude of the non-dominant leg show the opposite trend to the case of the dominant leg; i.e. the amplitude became smaller by bringing the opposite leg (dominant leg) closer to the tilt board's axis of rotation $(30 \pm 22,27 \pm 20$ and $22 \pm 18 \% \mathrm{MVC}$ for NS, MA and EA, respectively) (see Figure 2). But none of these decreases in the EMG amplitude reached the level of statistical significance $(p>0.05)$. The GM amplitude approached almost zero value when the SL (of the opposite/dominant leg) was used $(8 \pm 7$ $\% \mathrm{MVC}$ ). No statistically significant modulation of EMG average frequency was observed among WS, NS, MA, and EA. However, a significant decrease of the mean frequency was observed in the non-dominant leg during the SL stance. This resulted in statistically significant RANOVA values for the mean EMG frequency $(\mathrm{p}<0.05)$ of the non-dominant leg.

\section{Discussion}

The aim of this study was to make a comparison of GM activity during five balance tasks carried out on a tilt board that was inherently unstable in the frontal plane. The results of the study confirmed our hypothesis that asymmetrical parallel stance will cause increasingly more activity of the GM on that side of the body whose lever arm between the foot and the axis of rotation is shortened, and vice versa. The SL balance task generated the highest GM activity.

Little differentiation in GM amplitude between WS and NS stances was observed. This was probably because in these stances there is a sustained symmetrical loading of both legs, which stabilises the pelvis-hip complex in the frontal plane. It is one of the major functions of the GM to counter instability in such situations. Lastly, frequency analysis of the GM EMG activity showed no statistically significant differences among the five balance tasks when an observed leg was actively involved in the task. This suggests that there were no obvious differences among the tasks in motoneuron firing frequency.

Only a limited volume of literature regarding gluteal muscle activity during therapeutic exercises is 


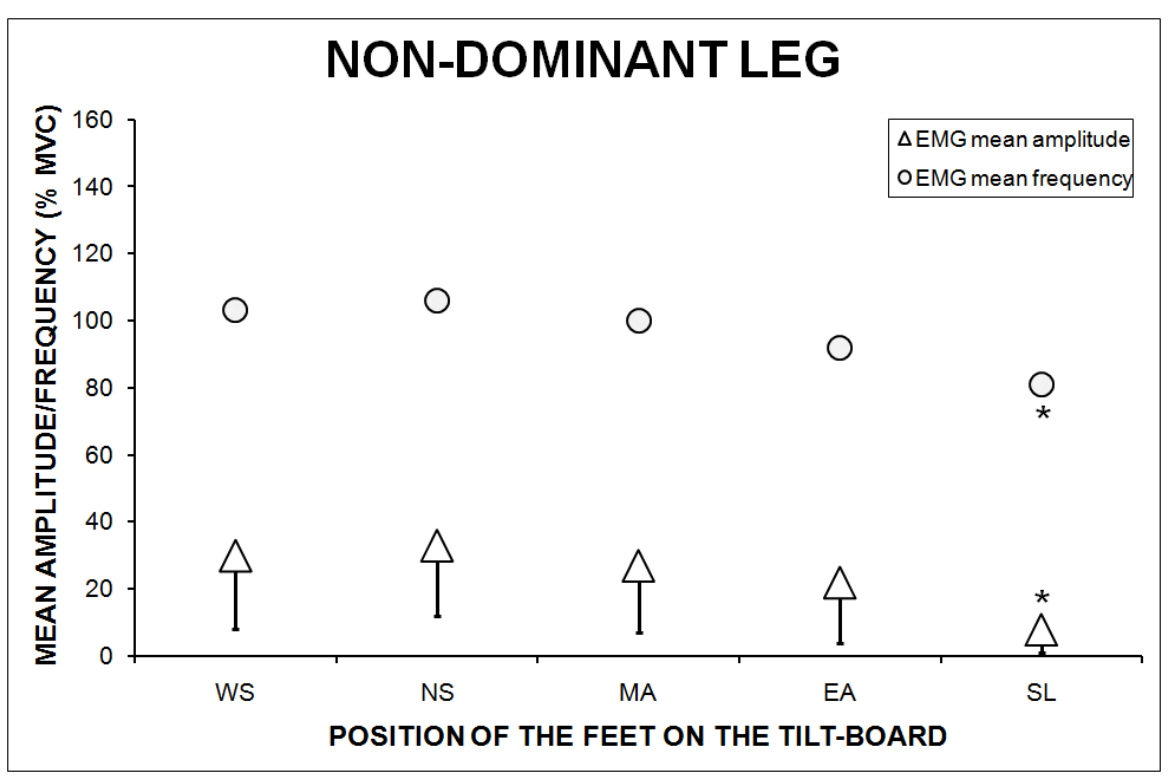

Fig 2. Results for the GM activity for the non-dominant leg during all the stances.

available for study $[1,8,14,16]$. Furthermore, the existing material is mostly oriented to those exercises that are typically used during the early stages of rehabilitation [3]. While information regarding muscle activation during these exercises is very important for clinical rehabilitation, the development of further knowledge about how the muscles function during more advanced exercises is crucial for developing techniques to apply during the later stages of rehabilitation and for injury prevention programs. Besides having an important role in locomotion, lunges, squatting, etc., GM is strongly involved in single leg weight bearing activities, especially when additional stabilization is required. During such activities GM is intensively active, but we need to advance slowly with this kind of functional loading. The results of our study show one way how this can be done. By shortening the lever arm of one foot relative to the axis of the tilt board, the weight bearing function of that leg can be stressed. This means that we can systematically increase/decrease the GM involvement under the functional conditions of a continuously unstable support surface. With this approach we can simulate, under laboratory conditions, the loadings which appear in many everyday activities and also in sports like alpine skiing for example. It is therefore very important that progressive stability/balance exercises be included in kinesiotherapeutic prevention and rehabilitation programs, whether the main focus of the program is on the hip itself or not.

The key role of strength and stability of the proximal body regions (trunk and pelvic girdle) has been pointed out several times. Much evidence exists which support the importance of strong and coordinated GM for the prevention of ankle, knee, hip and lower back injuries $[3,7,15,18]$. Significantly altered GM activation patterns have been observed under various conditions of local trauma/intervention [13] as well as that of other joints of lower extremity and lumbar spine. Moreover, different strengthening interventions that focussed on lumbo-pelvic muscle groups have been shown to reduce pain and improve function and biomechanics in the participating subjects [7]. Nevertheless, there is a lack of evidence about the effects of exercises which are designed to improve functional stability and balance. Such exercises are basically a combination of strength, power and neuromuscular coordination. Unfortunately, one aspect of the problem is that we have little knowledge about the intensity of these exercises.

Previous studies show that there are several ways by which the stimulation of the GM can be improved: (i) by changing the open kinetic chain exercises themselves [8]; (ii) by using the weight bearing exercises following the non-weight bearing exercises have been mastered $[1,8,14]$; or (iii) by asymmetrically increasing the load on the contralateral side while performing single leg stance exercises [19]. Practical experience and kinesiology evidence indicate that a combination of functional exercises that combine weight bearing stability and strength endurance requirements is the way to go in future programme design.

This concept was followed in the case of this study and the results revealed that by using different foot placements gradual increase in GM involvement can 
Hip abductors and unstable conditions

European Journal Translational Myology - Basic Applied Myology 2010; 1 (4): 187-192

be obtained, for example, when the foot was brought closer to the centre of rotation, while the other foot remained away from it, the GM activation was significantly increased.

To summarize, the results of this study point out the importance of studies of actual muscle functions by means of exercises that use a series of foot positions while balancing on a tilt board. In order to progressively load the lower extremity and to cause activity in hip side stabilizers, different degrees of positioning asymmetry should be used in practical training. We believe that the results of this study add to the understanding of the GM function under conditions that require a combination of weight bearing stability and strength endurance.

\section{Acknowledgements}

The authors would like to acknowledge the support of: (1) the EU Interreg-IVa project Mobility in Ageing (MOBIL - N_00033) of the Slovak-Austrian crossborder cooperation programme 2007-2013, and (2) the co-financing of the Austrian Federal Minister for Science and Research BM.W_F.

\section{Corresponding Author}

Nejc Sarabon, PhD, University of Primorska, Science and Research Center, Institute for Kinesiological Research, Garibaldijeva 1, SI 6000 Koper, Slovenia. Email: Nejc.sarabon@zrs.upr.si

\section{References}

[1] Ayotte NW, Stetts DM, Keenan G, Greenway EH. Electromyographical analysis of selected lower extremity muscles during 5 unilateral weight-bearing exercises. J Orthop Sports Phys Ther 2007;37(2):48-55.

[2] Beckman SM, Buchanan TS. Ankle inversion injury and hypermobility: effect on hip and ankle muscle electromyography onset latency. Arch Phys Med Rehabil 1995;76(12):1138-1143.

[3] Bolgla LA, Uhl TL. Reliability of electromyographic normalization methods for evaluating the hip musculature. J Electromyogr Kinesiol 2007;17(1):102-111.

[4] Brindle TJ, Mattacola C, McCrory J. Electromyographic changes in the gluteus medius during stair ascent and descent in subjects with anterior knee pain. Knee Surg Sports Traumatol Arthrosc 2003;11(4):244-251.

[5] Bullock-Saxton JE. Local sensation changes and altered hip muscle function following severe ankle sprain. Phys Ther 1994;74(1):17-28.

[6] Earl JE. Gluteus medius activity during 3 variations of isometric single-leg stance. Journal of Rehabilitation 2005;14(1):1-11.
[7] Earl JE, Hoch AZ. A Proximal Strengthening Program Improves Pain, Function, and Biomechanics in Women With Patellofemoral Pain Syndrome. Am J Sports Med 2010.

[8] Ekstrom RA, Donatelli RA, Carp KC. Electromyographic analysis of core trunk, hip, and thigh muscles during 9 rehabilitation exercises. J Orthop Sports Phys Ther 2007;37(12):754-762.

[9] Fredericson M, Cookingham CL, Chaudhari AM, et al. Hip abductor weakness in distance runners with iliotibial band syndrome. Clin J Sport Med 2000;10(3):169-175.

[10] Gottschalk F, Kourosh S, Leveau B. The functional anatomy of tensor fasciae latae and gluteus medius and minimus. $\mathrm{J}$ Anat 1989; 166:179-189.

[11] Grelsamer RP, McConnell J. The patella: a team approach. Aspen: Gaithersburg; 1998.

[12] Hart JM, Garrison JC, Kerrigan DC, PalmieriSmith R, Ingersoll CD. Gender differences in gluteus medius muscle activity exist in soccer players performing a forward jump. Res Sports Med 2007;15(2):147-155.

[13] Jacobs CA, Lewis M, Bolgla LA, et al. Electromyographic analysis of hip abductor exercises performed by a sample of total hip arthroplasty patients. J Arthroplasty 2009;24(7):1130-1136.

[14] Krause DA, Jacobs RS, Pilger KE, et al. Electromyographic analysis of the gluteus medius in five weight-bearing exercises. J Strength Cond Res 2009;23(9):2689-2694.

[15] Leavey VJ, Sandrey MA, Dahmer G. Comparative effects of 6-week balance, gluteus medius strength, and combined programs on dynamic postural control. J Sport Rehabil 2010;19(3):268-287.

[16] Mercer VS, Gross MT, Sharma S, Weeks E. Comparison of gluteus medius muscle electromyographic activity during forward and lateral step-up exercises in older adults. Phys Ther 2009;89(11):1205-1214.

[17] Mickelborough J, van der Linden ML, Tallis RC, Ennos AR. Muscle activity during gait initiation in normal elderly people. Gait Posture 2004;19(1):50-57.

[18] Nadler SF, Malanga GA, DePrince M, Stitik TP, Feinberg JH. The relationship between lower extremity injury, low back pain, and hip muscle strength in male and female collegiate athletes. Clin J Sport Med 2000;10(2):89-97.

[19] Neumann DA. Hip abductor muscle activity in persons with a hip prosthesis while carrying loads in one hand. Phys Ther 1996;76(12):1320-1330. 
Hip abductors and unstable conditions

European Journal Translational Myology - Basic Applied Myology 2010; 1 (4): 187-192

[20] Nyland J, Kuzemchek S, Parks M, Caborn DNM. Femoral anteversion influences vastus medialis and gluteus medius EMG amplitude: composite hip abductor EMG amplitude ratios during isometric combined hip abduction-external rotation. J Electromyogr Kinesiol 2004;14(2):255-261.

[21] Perry J. Pathologic gait. Instr Course Lect 1990;39:325-331.

[22] Rogers MW, Pai YC. Patterns of muscle activation accompanying transitions in stance during rapid leg flexion. J Electromyogr Kinesiol 1993;3(3):149-156.

[23] Schmitz RJ, Riemann BL, Thompson T. Gluteus medius activity during isometric closed-chain hip rotation. Journal of Sport Rehabilitation 2002;11(3):179-188.
[24] Sims KJ, Brauer SG. A rapid upward step challenges medio-lateral postural stability. Gait Posture 2000;12(3):217-224.

[25] Tirosh O, Sparrow WA. Age and walking speed effects on muscle recruitment in gait termination. Gait Posture 2005;21(3):279-288.

[26] Tyler TF, Nicholas SJ, Mullaney MJ, McHugh MP. The role of hip muscle function in the treatment of patellofemoral pain syndrome. Am J Sports Med 2006;34(4):630-636.

[27] Winter DA, Prince F, Frank JS, Powell C, Zabjek $\mathrm{KF}$. Unified theory regarding $\mathrm{A} / \mathrm{P}$ and $\mathrm{M} / \mathrm{L}$ balance in quiet stance. $\mathrm{J}$ Neurophysiol 1996;75(6):2334-2343.

[28] Zeller BL, McCrory JL, Kibler WB, Uhl TL. Differences in kinematics and electromyographic activity between men and women during the single-legged squat. Am J Sports Med 2003;31(3):449-456. 